\title{
Measurement of Aerodynamic and Acoustic Quantities Describing Flow around a Body Placed in a Wind Tunnel
}

\author{
Miroslav Mahdal ${ }^{1}$, Josef Dobeš ${ }^{2}$, Milada Kozubková ${ }^{3}$ \\ ${ }^{I}$ The Department of Control Systems and Instrumentation, Faculty of Mechanical Engineering, Technical University of \\ Ostrava, 17. listopadu 15, 708 33, Ostrava, Czech Republic, miroslav.mahdal@vsb.cz \\ ${ }^{2}$ Hanon Systems Autopal Services, s. r. o., Lužická 984/14, 741 01, Nový Jičín, Czech Republic, jdobes@hanonsystems.com \\ ${ }^{3}$ Department of Hydromechanics and Hydraulic Equipment, Faculty of Mechanical Engineering, VŠB - Technical University \\ of Ostrava, 17. listopadu 15, 70833 Ostrava - Poruba, Czech Republic, milada.kozubkova@vsb.cz
}

\begin{abstract}
Aerodynamically generated noise affects passenger comfort in cars, high-speed trains, and airplanes, and thus, automobile manufacturers aim for its reduction. Investigation methods of noise and vibration sources can be divided into two groups, i.e. experimental research and mathematical research. Recently, owing to the increase in computing power, research in aerodynamically generated noise (aero-acoustics) is beginning to use modern methods such as computational fluid dynamics or fluid-structure interaction. The mathematical model of turbulent flow is given by the system of partial differential equations, its solution is ambiguous and thus requires verification by physical experiment. The results of numerical methods are affected by the boundary conditions of high quality gained from the actual experiment. This article describes an application of complex measurement methodology in the aerodynamic and acoustic (vibro-acoustic) fields. The first part of the paper is focused on the specification of the experimental equipment, i.e. the wind tunnel, which was significantly upgraded in order to obtain the relevant aerodynamics and vibro-acoustics data. The paper presents specific results from the measurement of the aerodynamic and vibroacoustic fields.
\end{abstract}

Keywords: Aerodynamic, acoustic, wind tunnel, cylinder, sphere, computational fluid dynamics.

\section{INTRODUCTION}

Owing to the increasing speed of modern vehicles and the reduced noise of the driving system, aerodynamic noise becomes the dominant noise component, which negatively affects not only passengers but also the surrounding environment.

The research work published by Z. Sun and colleagues [1] reported on the practical application of the methodology of the numerical simulation flow around the high-speed train, i.e. the prediction of generated noise from the train itself and the subsequent distribution of acoustic waves in the vicinity, together with experimental measurements. The nonlinear acoustics solver (NLAS) method and the approach of computation of the FW-H for the noise source, proposed by Ffowcs-Williams and Hawkings, are used to solve the problem. In this paper, the actual measurement results are reported, which are in good agreement with the CFD (computational fluid dynamics) results. The study shows that the noise at a defined distance, at a speed of $300 \mathrm{~km} \cdot \mathrm{h}^{-1}$, is $80 \mathrm{~dB}(\mathrm{~A})$. The probe for the noise measurement was located $25 \mathrm{~m}$ from the side of the train and $3 \mathrm{~m}$ above the track.
Probably the largest application area of the measurement and modeling of acoustic and aero-acoustic phenomena is in the automotive industry, in both the internal and external aeroacoustics of cars. A study on intra-vehicle aeroacoustics could include the optimization of the heating, ventilation, and air conditioning (HVAC) of the car itself. Examples of studies on the optimization of the flow of air from the air conditioner, through the cabin and the dashboard spaces, can be the works of S. Détry and J. Manera [2], [3]. From their work, the procedure for acoustic noise measurement and validation of the experimentally obtained data with numerical results are described. The measurement is made using a microphone at a distance of $1 \mathrm{~m}$ from the air-conditioning output located on the dashboard. In this paper, a dual approach to the numerical modeling is presented - airconditioning outlets without and with the regulating flaps at the end of the pipeline. The influence of the regulating flaps on the noise generated by the ventilation is evaluated. Using experimental measurements, the greatest sources of the noise were found - here it was the $90^{\circ}$ bend of the pipeline for fluid flow. This source problem was subsequently subjected to numerical simulations to obtain the optimized output. 
The optimization may also apply to other interior parts of the vehicle, such as the fuel line for diesel engines, as stated in the work by B. Van Antwerpen [4]. In this article, the aeroacoustic and vibro-acoustic modeling are combined. High pressures in the pipeline for the transport of diesel into the combustion chamber cause vibrations and consequently, acoustic waves propagate from these vibrations. The validation of the experimentally obtained data and CFD simulation was performed for two temperatures. The optimization reduced the noise level by more than $10 \mathrm{~dB}$. The external aeroacoustics of cars include the respective numerical modeling of the following elements: A-pillars (its shape and complexity - sealing rubber, etc.), side mirror, and side window. It is possible to Find solutions for all three elements simultaneously, however, this already requires enormous demands on computing mesh and geometry. A contribution by M. Gustafsson [5] includes complex modeling of acoustic phenomena, i.e. the entire area around a Volvo truck was numerically simulated in this case. As a source, the engine was modeled at this point; subsequently, deformation and propagation of acoustic waves through the other parts of the truck such as the engine compartment, cabin, and chassis were examined. Numerical modeling revealed three main sources of noise: the cylinder block (noise level $\sim 70 \mathrm{~dB}(\mathrm{~A})$ ), gearbox $(\sim 65 \mathrm{~dB}(\mathrm{~A}))$, and cylinder head $(\sim 60 \mathrm{~dB}(\mathrm{~A}))$. Validation of the simulations and the experiment was performed for three engine speed settings $\left(600 \mathrm{~min}^{-1}, \quad 1200 \mathrm{~min}^{-1}\right.$, and $\left.1900 \mathrm{~min}^{-1}\right)$, and these comparisons were evaluated as the dependent sound pressure level vs. frequency.

The experimental measurement and modeling of the noise generated by the A-pillar itself has been dealt with by many authors [6], [7]. CFD analysis is applied to the shape of the A-pillar itself and the windshield sealing rubber. Measurements are made at different fixed locations, and these measurements are compared to the results of the numerical simulations. From the four tested profiles the most suitable one, owing to the numerical simulation, is the one with the lowest noise level.

The side rearview mirror of a car has been investigated in many studies [8], [9], [10], [11]. These studies focused on the problem of flow around solutions, which define the acoustic noise sources and the numerical modeling of acoustic wave propagation. The research work is complemented by experiments and evaluation of quantities that describe acoustic wave propagation phenomena. Combining the Apillar and side rearview mirrors has been the focus of many authors [12], [13]. The interaction between the turbulence structures from the A-pillar and the side mirror has been understood [13], and various shapes and dimensions of the Apillar have been tested to achieve a noise level reduction. Two versions of both, the A-pillar and the side mirror, as well as combinations of both, were tested, making a total of four tested variants. The measurements were made on the driver's side window and the distribution of acoustic sources and pressure fluctuations was compared. The experimental measurement, for which 96 miniature microphones from the outside were attached to the side window, is reported in the paper. The evaluation was performed using the statistical energy analysis (SEA) methodology. The pressure fluctuations and the wave spectrum around the noise source were evaluated using graphical means. For future research, these extensive reference sources can be used for numerical modeling and experimental measurements of acoustic wave generation in the wind tunnel on actual car components. Methods of measuring and processing noise and vibrations and also numerical methods are also described in articles [16], [17], [18], [19].

The measurement methodology proposed in this paper is a comprehensive experimental method for complex aeroacoustic problems, which are increasingly required in the field of virtual prototyping using computer-aided engineering (CAE) methodology. In this research field (CAE), with the help of numerical simulations such as CFD - the sound level is reduced by optimizing the shape of a given part at no cost and in no time to produce each prototype and carry out its subsequent testing. As mentioned before, at least one experimental measurement of an actual prototype of a previously studied element is always necessary to enable the verification of the correctness of the mathematical model setup and, additionally, for the redefinition of the boundary conditions of mathematical simulations.

Measurement of turbulent flow, noise and vibration variables requires the use of quality measuring devices and evaluation methods. To verify the measurement methodology of aerodynamic and vibro-acoustic properties of flow around the object in a wind tunnel, single test bodies were selected, e.g., a cylinder or a sphere, as previous studies have already focused on them. The results are processed in such a way that they can serve as boundary conditions as well as verification examples for the mathematical modeling of turbulent flow characterized by the vortex shedding behind the obstacle extended by the modeling of turbulence-induced noise.

\section{EXPERIMENTAL DEVICES}

For the aerodynamic field measurement, the modern method involving the use of a hot-wire anemometer MiniCTA (constant temperature anemometry) manufactured by Dantec Dynamics $\mathrm{GmbH}$ was used. The velocity measurement range was $0.01 \div 500 \mathrm{~m} \cdot \mathrm{s}^{-1}$, maximum vortex shedding frequency up to $400 \mathrm{kHz}$. The microphone type 4190 - Free-field 1/2"-microphone with a nose cone adapter type UA0386 from Brüel \& Kjaer was used to measure the sound pressure level. The frequency range of the measuring microphone is $1 \div 40 \mathrm{kHz}$. The nose cone allows measurement using a microphone in the wind tunnel where its shape directs the airflow around the microphone such that there is no additional noise in the measured data due to the flow through the microphone grid. For the vibration measurement, the portable vibrometer OMETRON VH-1000-D was used. The frequency range of the measuring device is $0.5 \div 22 \mathrm{kHz}$. The measurement principle consists of a frequency shift in the high-frequency carrier signal due to the Doppler effect. For the evaluation of the aerodynamic field, the time-averaged velocity and turbulence intensity profiles were used. For the vibro-acoustic part of the measurement, the FFT evaluation method was used for the graphical representation of the obtained values. 
The experimental device (Fig.1.) comprises a settling chamber, honeycomb and screens, contraction cone, test section, diffuser and fan with noise silencers, and electrical accessories (frequency converter, traverser control, power supply of the hot wire anemometer), along with the possibility of adding a fog generator (flow visualization). The air flow direction in the wind tunnel is depicted by the white arrow.

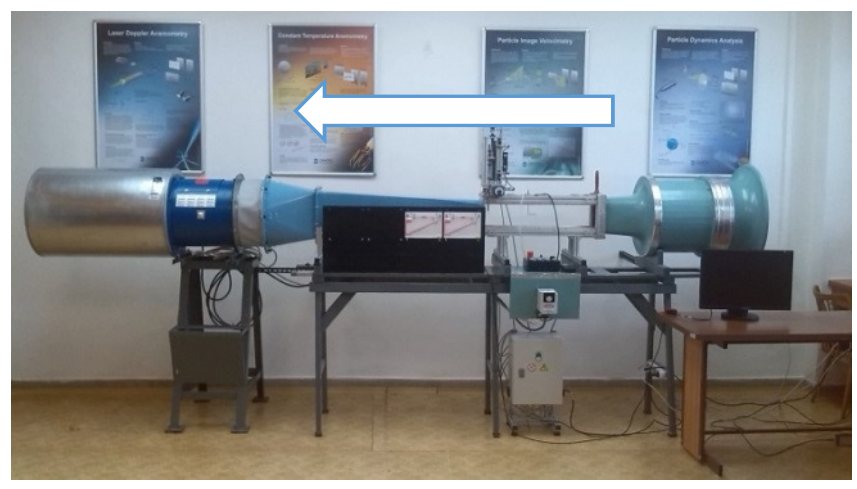

Fig.1. Experimental wind tunnel - open design (Eiffel type).

\section{EXPERIMENTAL MEASUREMENT OF AERODYNAMIC FIELD BEHIND THE CYLINDER}

The velocity profiles were measured at the entrance to the measuring section and behind the body placed in the flow, according to the schematic shown in Fig.2., by using the MiniCTA hot-wire anemometer methodology - probe 55P14. To calculate the boundary conditions of the mathematical model, it is necessary to perform measurements at the input to the measuring section (marked as "VELOCITY PROFILE AT THE START" in Fig.2.).

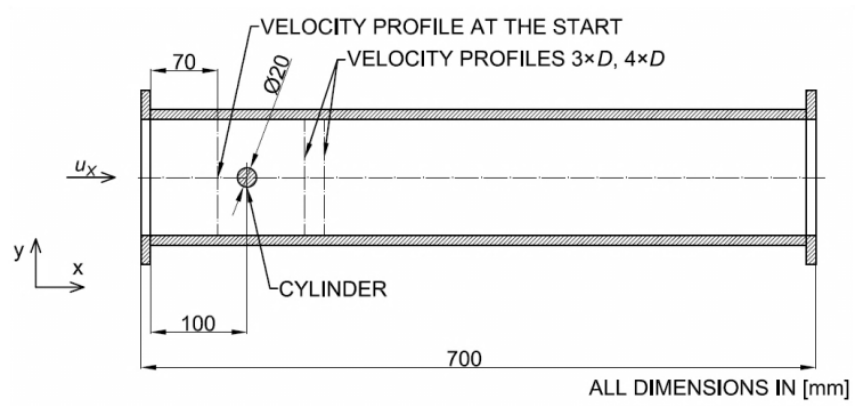

Fig.2. Aerodynamic flow field scheme measurement - the horizontal cut of the measuring section.

The measurement parameters are the sampling frequency $f_{S R}=20 \mathrm{kHz}$ and the number of measured samples $N=65536$. The obtained result of the measurement is the time-averaged velocity component $u_{S X}$ at the input of the measuring section, which is graphically presented in Fig.3. Due to the construction of the wind tunnel, only half the flow characteristics were measured. For CFD numerical simulation purposes, the assumption of profile symmetry was considered. Using the MiniCTA program, the time-averaged velocity $u_{S X}$ measured data were processed as the timeaveraged intensity turbulence profile $I_{T S X}$. The graphical representation of the intensity turbulence $I_{T S X}$ can be seen in Fig.4., and the equation for the calculation of the turbulence intensity is given in the literature [14].

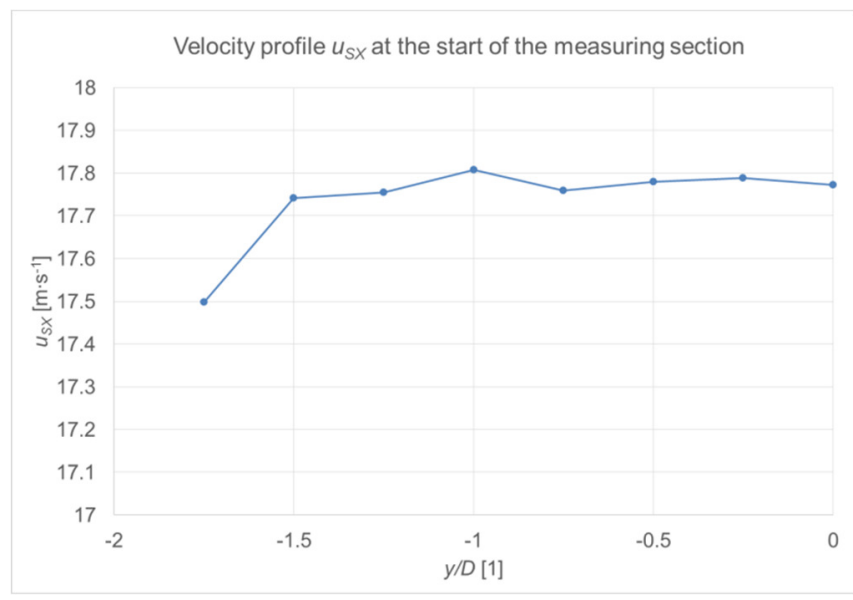

Fig.3. Velocity profile at the start of the measuring section of the wind tunnel with the inserted cylinder.

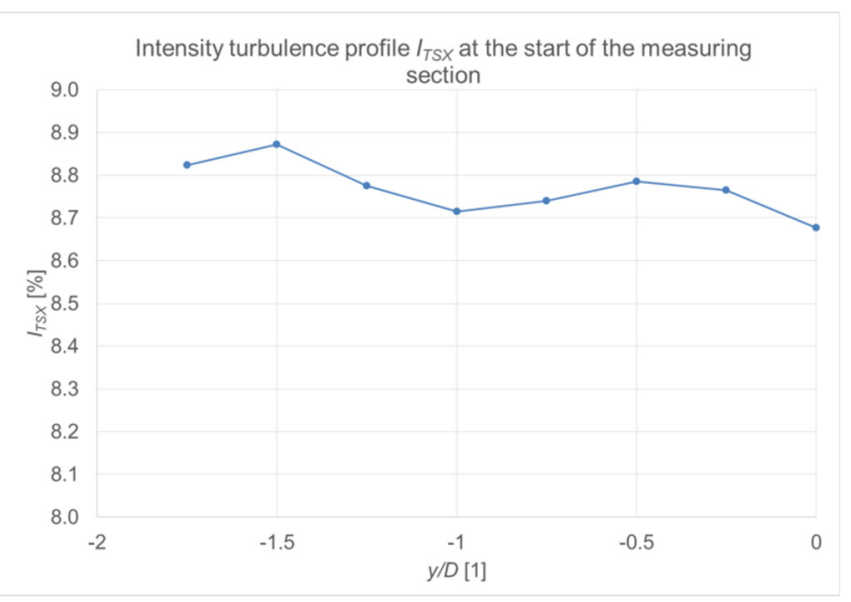

Fig.4. Intensity turbulence profile at the start of the measuring section of the wind tunnel with the inserted cylinder.

In the graphs, the velocity values $u_{S X}$ and turbulence intensity values $I_{T S X}$ are plotted on the vertical axes, and the horizontal axis represents the relative distance value $y / D$, where $D$ denotes the cylinder diameter $D=d_{V}=0.02 \mathrm{~m}$. Coordinate $y$ denotes the coordinate axis in Fig.2. The value $y / D=0$ indicates the initial position in the axis of the flow around the cylinder. The minus sign marks the right half of the wind tunnel from the point of view of entrance velocity (Fig.2.). From the measurement, the quantities are obtained for calculating boundary conditions that are needed to solve the mathematical problem of the CFD code using the computational programs OpenFOAM and ANSYS Fluent, using velocity $u_{S X}=17.8 \mathrm{~m} \cdot \mathrm{s}^{-1}$ and turbulence I ntensity $I_{T S X}=8.75 \%$. The intensity of the turbulence is higher than 
that stated in the literary sources [14]. The accuracy of measurement by the hot-wire anemometer and the subsequent evaluation of the $I_{T}$ turbulence intensity was verified considering the air flow in the circular pipeline. This higher $I_{T S X}$ turbulence intensity is due to the tunnel input geometry in the measuring section.

In Fig.5. the graphical results of experimental measurement of dimensionless velocity profiles are plotted behind the cylinder placed in the flow at a distance of $3 \times D$ and $4 \times D$. In Fig.6. the graphical results of the dimensionless turbulence intensity at a distance of $3 \times D$ and $4 \times D$ are plotted. The dimensionless profiles are obtained by dividing the measured value (velocity $u_{X}$ and turbulence intensity $I_{T X}$ ) by the value obtained by measuring at the beginning of the section $\left(u_{S X}\right.$, $\left.I_{T S X}\right)$.

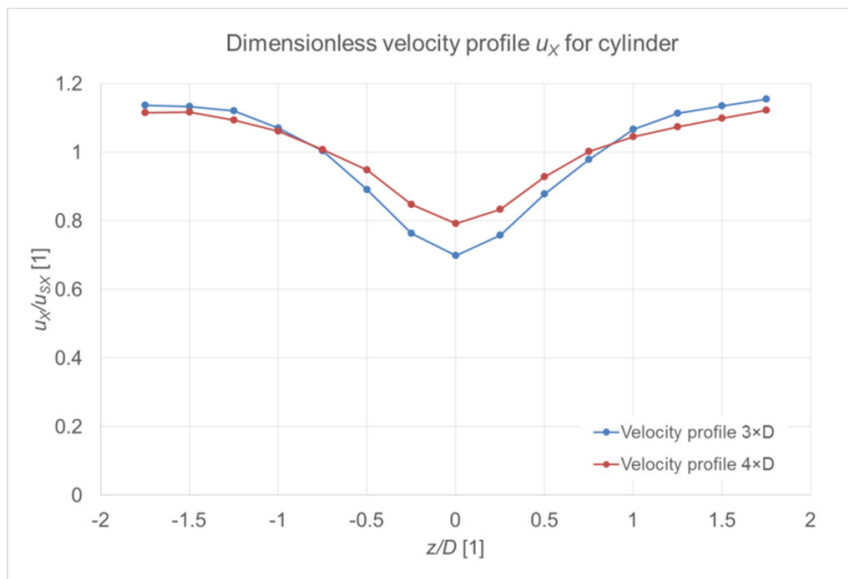

Fig.5. Dimensionless velocity profiles behind the cylinder placed in the flow.

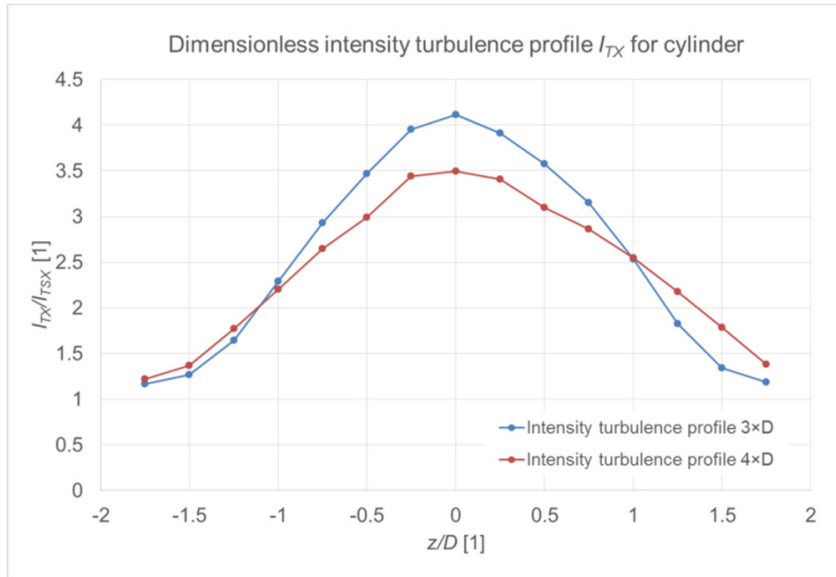

Fig.6. Dimensionless intensity turbulence profiles behind the cylinder placed in the flow.

These experimentally acquired time-averaged dimensionless profiles (velocity $u_{X}$ and turbulence intensity $\left.I_{T X}\right)$ are used to verify the aerodynamic part of the task computed from the numerical simulations by the computational programs OpenFOAM and ANSYS Fluent.

\section{EXPERIMENTAL MEASUREMENT OF AERODYNAMIC FIELD BEHIND THE SPHERE}

Velocity profiles were measured by the same method as for the cylinder. The aerodynamic flow field scheme for the sphere is shown in Fig. 7.

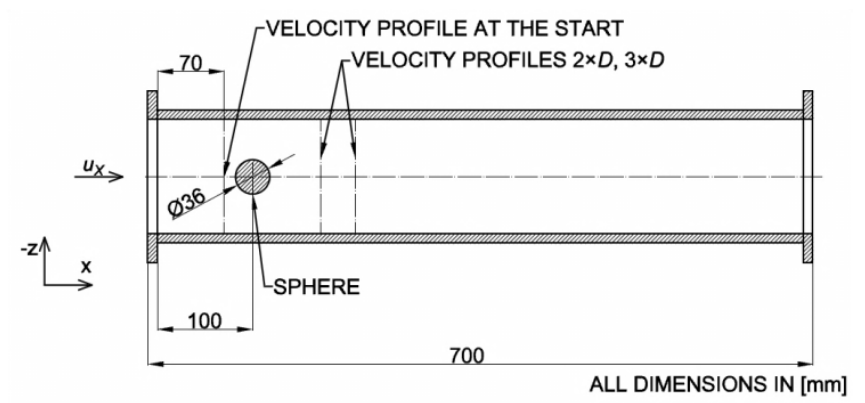

Fig.7. Aerodynamic flow field scheme measurement for the sphere.

We use also the same measurement method for calculating the boundary conditions of the mathematical model. The obtained result of the measurement is the time-averaged velocity component $u_{S X}$ at the input of the measuring section, which is graphically presented in Fig.8.

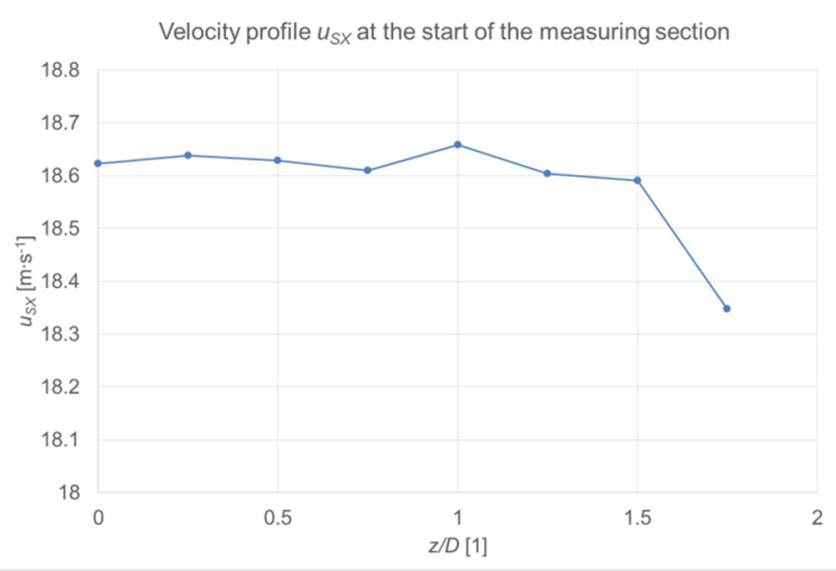

Fig.8. Velocity profile at the start of the measuring section of the wind tunnel with the inserted sphere.

The graphical representation of the intensity turbulence measurement results $I_{T S X}$ can be seen in Fig.9. In the graphs, the velocity values $u_{S X}$ and turbulence intensity values $I_{T S X}$ are plotted on vertical axes. On the horizontal axis is the distance relative value $z / D$, where $D$ denotes the sphere diameter $D=d_{K}=0.036 \mathrm{~m}$. The coordinate $z$ denotes the coordinate axis shown in Fig.7. The value $z / D=0$ indicates the initial position in the axis of the sphere placed in the flow. The plus sign marks the right half of the wind tunnel from the point of view of entrance velocity (Fig.7.). The boundary conditions, for the CFD code for the sphere task, are velocity $u_{S X}=18.65 \mathrm{~m} \cdot \mathrm{s}^{-1}$, turbulence intensity $I_{T S X}=8 \%$. Equivalent results of the verification for circular pipeline, as in the case of the cylinder, were obtained. Furthermore, the assumption of the profile symmetry at the entrance to the measuring section was also applied. 


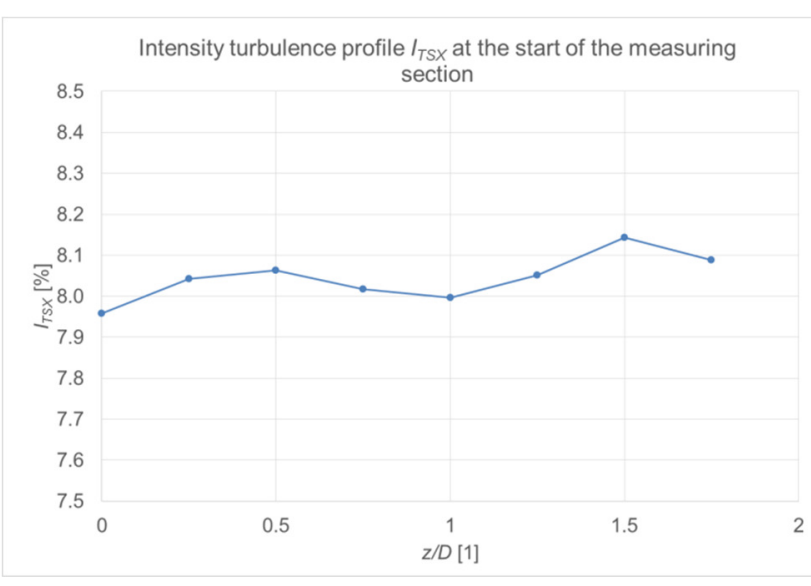

Fig.9. Intensity turbulence profile at the start of the measuring section of the wind tunnel with the inserted sphere.

In Fig.10. the graphical results of experimental measurement of the dimensionless velocity profiles are plotted behind the sphere placed in the flow at a distance of $2 \times D$ and $3 \times D$. In Fig. 11 . the graphical results of the dimensionless turbulence intensity at a distance of $2 \times D$ and $3 \times D$ are plotted. The dimensionless profiles are obtained by the same procedure as for the cylinder task.

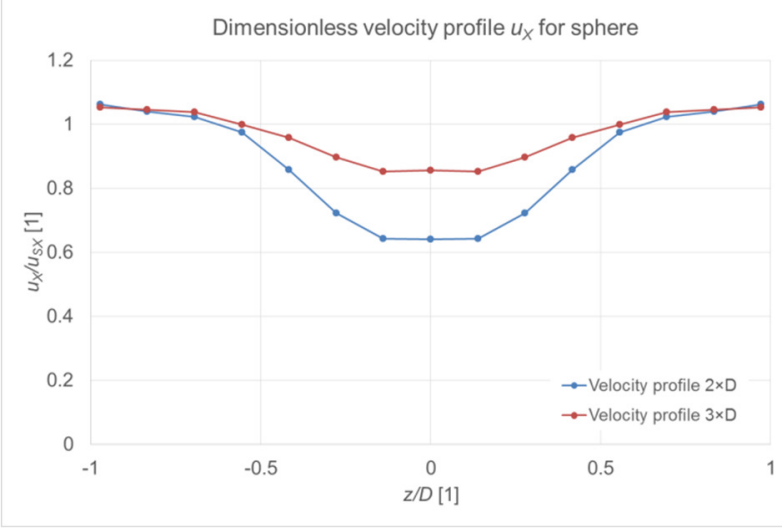

Fig.10. Dimensionless velocity profiles behind the sphere placed in the flow.

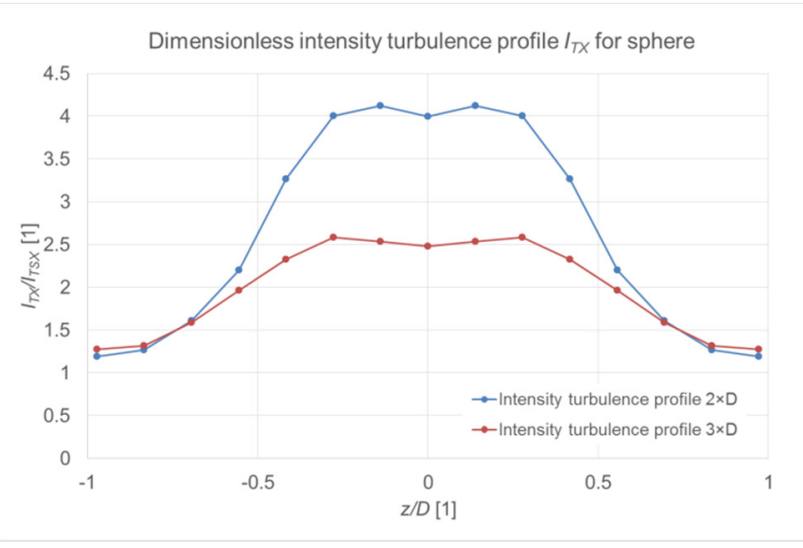

Fig.11. Dimensionless intensity turbulence profiles behind the sphere placed in the flow.

\section{EXPERIMENTAL MEASUREMENT OF VIBRO-ACOUSTIC PART} FOR THE CYLINDER TASK

The measurement characteristics of the vibro-acoustic field - i.e., sound pressure level $S P L$ and vibration - are shown in Fig.12. For measurement of the sound pressure level $S P L$, the microphone with nose cone $1 / 2$ " (Brüel \& Kjaer) was used, and a non-contact laser vibrometer was used for vibration sensing.

The sampling frequency of the pulse analyzer for the presentation of the results (Fig.13., Fig.14.) was set at the value $f=6.4 \mathrm{kHz}$. For the measurements, the $S P L$ and vibration of the empty wind tunnel at the sampling frequency $f=12.8 \mathrm{kHz}$ were also performed. However, these measurements are not presented here, as they only serve as a benchmark for comparing with the following vibro-acoustic measurements of the wind tunnel with the wrapped cylinder.

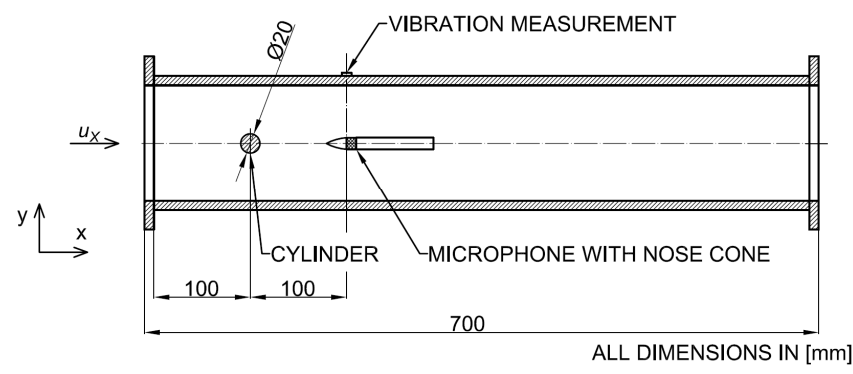

Fig.12. Vibro-acoustic flow field scheme measurement - the horizontal cut of the measuring section.

The results of the sound pressure level $S P L$ measurement of the empty wind tunnel are shown in Fig.13. The $S P L$ value is calculated using the following equation, which is based on the reference pressure $p_{R E F}=2 \cdot 10^{-5} \mathrm{~Pa}$ :

$$
\mathrm{SPL}=20 \cdot \log _{10}\left(\frac{p_{\mathrm{RMS}}^{\prime}}{p_{\mathrm{REF}}}\right)
$$

$p_{R M S}^{\prime}$ in the equation means root-mean-square of the fluctuation pressure $p$. In Fig. 13. the data for the measurement with a nose cone and without a nose cone are compared. The results show that the measured quantities are the same for both cases of microphone arrangement and that a nose cone is not necessary for this type of measurement. However, it was used in all measurements in accordance with the recommendations of the equipment supplier and the nature of the experiment.

Fig.14. is a graphical representation of the vibration for an empty tunnel measurement, with the curves depicting the results using a microphone with/without a nose cone. The output of the measured vibration signal from the OMETRON VH-1000-D laser sensor is velocity, so the vibration results are displayed as the velocity ratio in decibels. To evaluate the vibration, a modified form of equation (1) is used, where the pressure quantity $p$ is changed for the velocity quantity $u$, and the reference velocity value is $u_{R E F}=1 \cdot 10^{-6} \mathrm{~mm} \cdot \mathrm{s}^{-1}$. The results do not differ, because the vibration sensing is unaffected by the presence or absence of a nose cone on the microphone. 


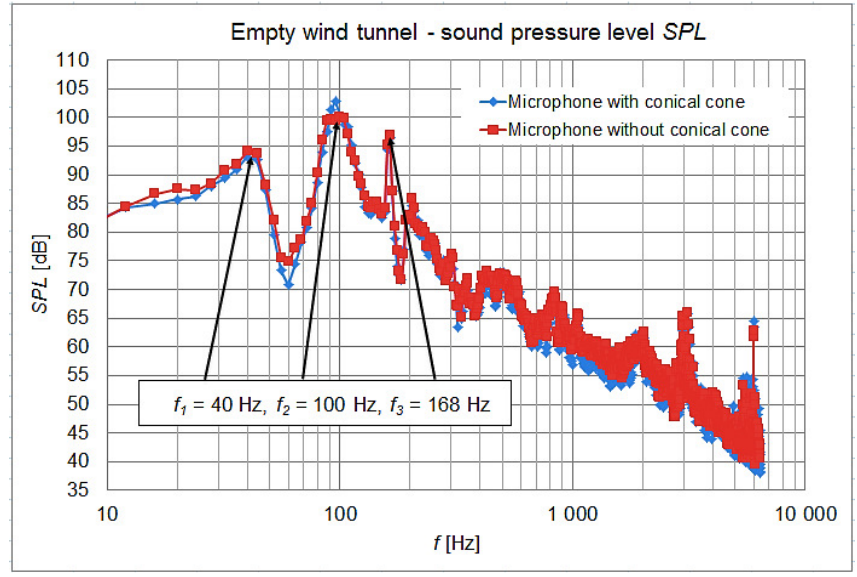

Fig.13. SPL experimental results of the empty wind tunnel.

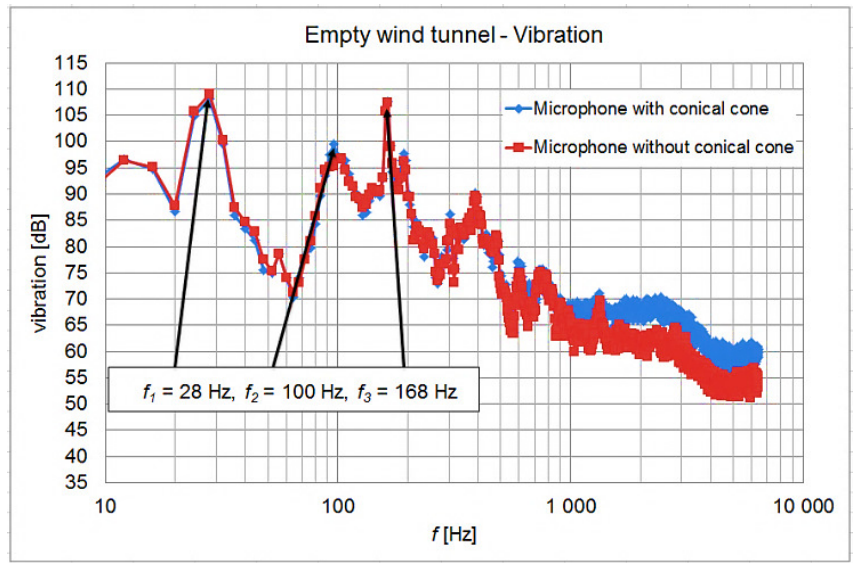

Fig.14. Vibration measurement results of the empty wind tunnel.

The graphical results of the $S P L$ and vibration show significant $f_{1}, f_{2}$, and $f_{3}$ frequencies. Frequency $f_{1}$ $(S P L=40 \mathrm{~Hz}$; vibration $=28 \mathrm{~Hz})$ can be assigned to the frequency obtained when the frequency converter is set to $f_{F M}=28 \mathrm{~Hz}$ for all measurements, which controls the wind tunnel fan speed. The frequency shift at the SPL measurement is caused by the acoustic signal passage generated by the frequency converter through the transparent Plexiglas of the measuring section into the wind tunnel measurement area where $S P L$ is measured by the microphone. Frequency $f_{2}$ $(S P L=100 \mathrm{~Hz}$; vibration $=100 \mathrm{~Hz})$ is two times greater than the power supply frequency and it is not possible to fully confirm that the electricity frequency is reflected in the measurement because it can be the natural frequency of the experimental device itself when the air flows at the velocity set by frequency $f_{F M}=28 \mathrm{~Hz}$. Frequency $f_{3}(S P L=168 \mathrm{~Hz}$; vibration $=168 \mathrm{~Hz}$ ) corresponds to twice the value of the Blade Pass Frequency ( $B P F)$ of the wind tunnel fan, which is calculated by the equation:

$\mathrm{BPF}=\frac{30 \cdot f \cdot b}{60}=\frac{30 \cdot 28 \cdot 6}{60}=84 \mathrm{~Hz} \rightarrow 84 \cdot 2=168 \mathrm{~Hz}$ where $f$ is the frequency set by the frequency converter, parameter $b$ indicates the number of fan blades, and the number 30 is the fan constant from the measured linear dependence of the frequency on the wind tunnel fan speed. The resulting measured frequency is, therefore, the "exposure" of the fan speed to the measurement results.

From the graph in Fig.15. the experimental results of the $S P L$ for the wind tunnel without an inserted object are plotted - the red curve; the measurement results of the $S P L$, for the flow around the cylinder in the wind tunnel, are plotted as the blue curve. From these results, it can be seen that the SPL value for the flow around the cylinder is higher when the first peak is at the frequency $f_{E X P 1}=192 \mathrm{~Hz}$ and noise value $S P L_{E X P 1}=102 \mathrm{~dB}$. The second peak for the flow around a cylinder experimentally obtained the parameters $f_{E X P 2}=380 \mathrm{~Hz}$ and $S P L_{E X P 2}=99 \mathrm{~dB}$.

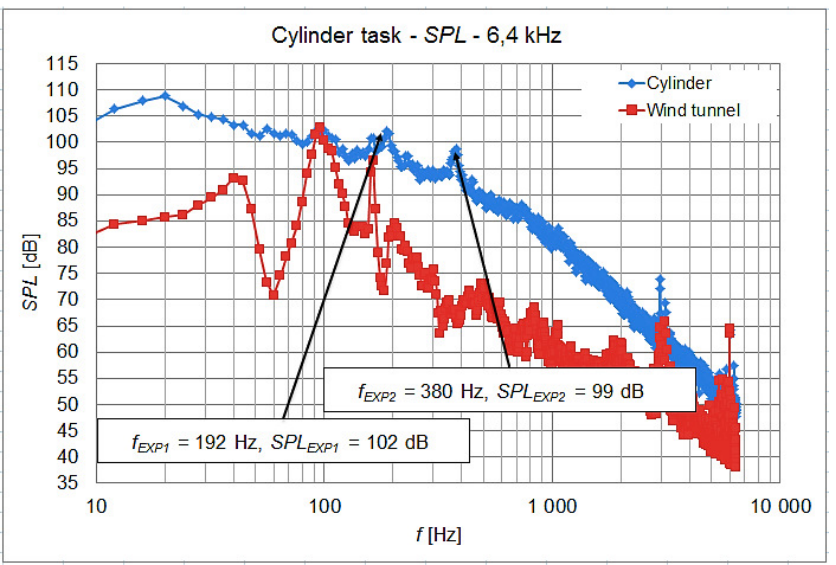

Fig.15. SPL experimental results for flow around the cylinder.

The results of the experimental vibration (Fig.16.) reveal a smaller peak at the frequency $f_{E X P I}=188 \mathrm{~Hz}$ with effective vibration value of $103 \mathrm{~dB}$. The second peak is noted at the frequency $f_{E X P 2}=380 \mathrm{~Hz}$ with the effective vibration value of $93 \mathrm{~dB}$. No significant differences between the empty wind tunnel and the flow around a cylinder are visible from the vibration measurement results. This may be due to the wooden support structure of the wind tunnel, which may not be sufficiently stiff.

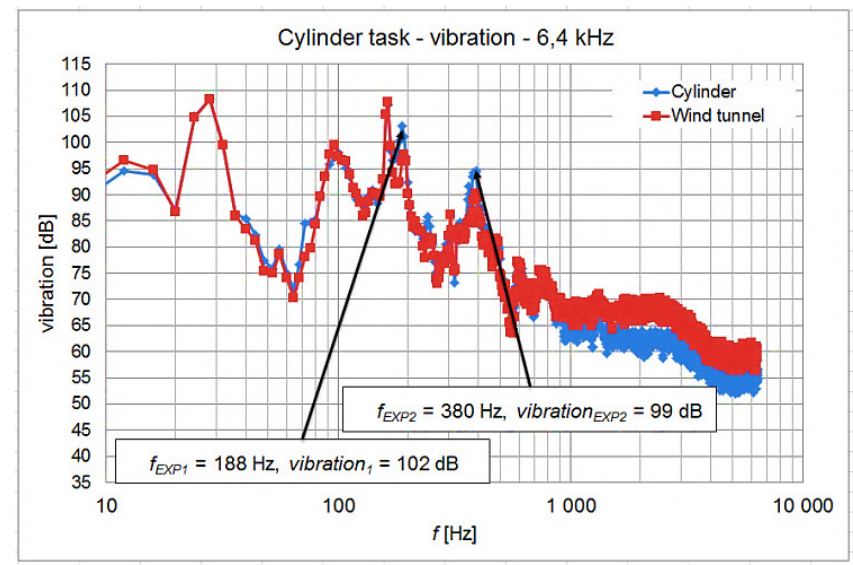

Fig.16. Experimental results of vibrations for flow around the cylinder. 
In chapters 2 and 4, the experimentally obtained data at the measuring aero-acoustic task for the cylinder are presented. In this section, the focus is on the aerodynamic field. The velocity profiles $u_{S X}$ (Fig.3.) and turbulence intensity $I_{T S X}$ (Fig.4.) are measured at the beginning of the measuring section, as shown in Fig.2. These measurements are used to calculate the boundary conditions of numerical simulations using the finite volume method by means of selected computational programs OpenFOAM and ANSYS Fluent. Furthermore, the dimensionless velocity profiles $u_{X}$ (Fig.5.) and dimensionless turbulence profiles $I_{T X}$ (Fig.6.) are evaluated at a distance $3 \times D$ and $4 \times D$, as shown in Fig. 2 . These graphs, describing the aerodynamic flow field behind the flow around a cylinder, can be compared to the results obtained by the selected turbulent models obtained through the numerical tools OpenFOAM and ANSYS Fluent on the same computing numerical mesh.

The results corresponding to the vibro-acoustic measurement field, i.e. the SPL measured by the microphone with nose cone (Fig.12.), can be used to verify the numerical simulations obtained through acoustic analogies such as Curle's analogy (OpenFOAM) and Ffowcs-Williams and Hawking's analogy (ANSYS Fluent). The selected turbulent models will be compared to the experimental results. The presented results from vibration measurements, using the proximity laser vibrometer (Fig.16.), will be used for future research in numerical simulations by means of the "fluid structure interactive", using the OpenFOAM and ANSYS Fluent/Mechanical. The methodology thus developed for aero-acoustic transmission and vibration generation on a fixed surface can be applied to the real task of determining the impact of acoustic waves on passengers in a car from the rearview mirror of the car.

\section{EXPERIMENTAL MEASUREMENT OF VIBRO-ACOUSTIC FIELD FOR FLOW FIELD AROUND THE SPHERE}

Just as for the cylinder, the vibro-acoustics field, SPL, and vibration were measured for the sphere (Fig.17.).

For the obtained quantities describing the vibro-acoustic behavior of the empty tunnel, a basic measurement corresponding to Fig.17. was performed without an inserted sphere. The results for the empty tunnel are presented in the previous chapter on the cylinder task. Using equation (3) for the Strouhal number $S t$, the vortex shedding frequency $f_{V S}$ for the flow around the sphere is determined:

$$
\mathrm{St}=\frac{f_{\mathrm{VS}} \cdot l}{u_{\mathrm{SX}}} \rightarrow f_{\mathrm{VS}}=\frac{u_{\mathrm{SX}} \cdot \mathrm{St}}{l}=\frac{18.65 \cdot 0.193}{0.036}=99.98 \mathrm{~Hz}
$$

where $\mathrm{l}$ is the diameter of the sphere $d_{K}=0.036 \mathrm{~m}, u_{S X}$ is the input speed, $u_{S X}=18.65 \mathrm{~m} \cdot \mathrm{s}^{-1}$, and $S t$ is the Strouhal number for the sphere with specified Reynolds number $R e=43711$. From Fig.18. for the peak frequency $f_{V S}=99.98 \mathrm{~Hz}$ is determined, and at the frequency $f_{E X P I}=100 \mathrm{~Hz}$ is the sound pressure level $S P L_{E X P I}=96 \mathrm{~dB}$. Vibration measurement results (Fig.19.) show a lower peak at $f_{E X P I}=100 \mathrm{~Hz}$ and an effective vibration value of $92 \mathrm{~dB}$, which is the closest calculated value of the vortex shedding frequency
$f_{V S}=99.98 \mathrm{~Hz}$. Frequency $f_{2}(S P L=162 \mathrm{~Hz}$; vibration $=$ $164 \mathrm{~Hz}$ ) corresponds to twice the value of the so-called Blade Pass Frequency (BPF) of the tunnel fan (see equation (2)). Measurement of the sound pressure level SPL (Fig.18.) and vibrations (Fig.19.) is performed for the sampling frequency of the pulse analyzer $f=1.6 \mathrm{kHz}$.

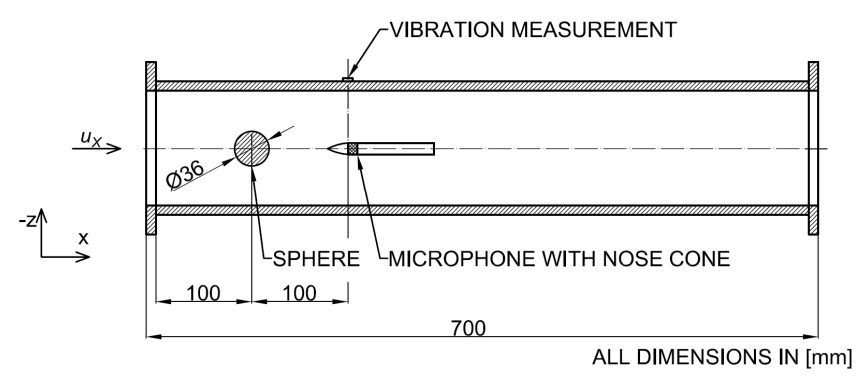

Fig.17. Vibro-acoustic flow field measurement scheme for the sphere - the horizontal cut of the measuring section.

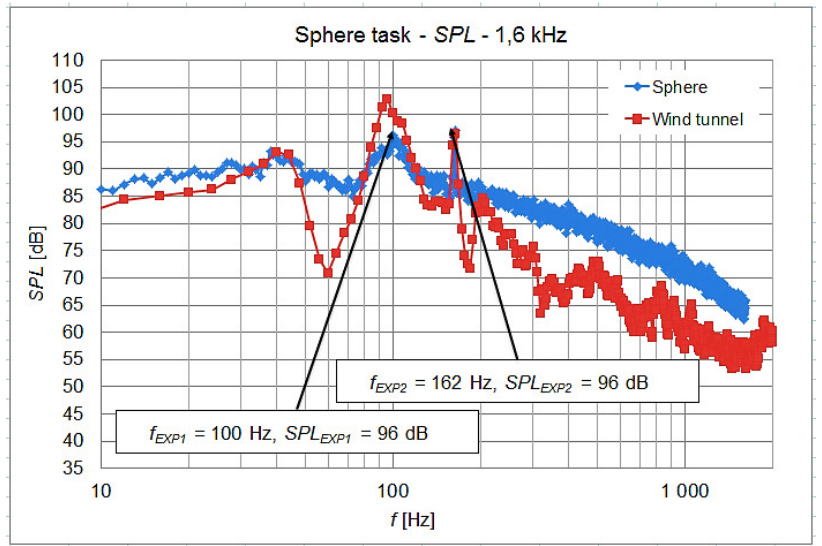

Fig.18. SPL measurement results for flow around the sphere.

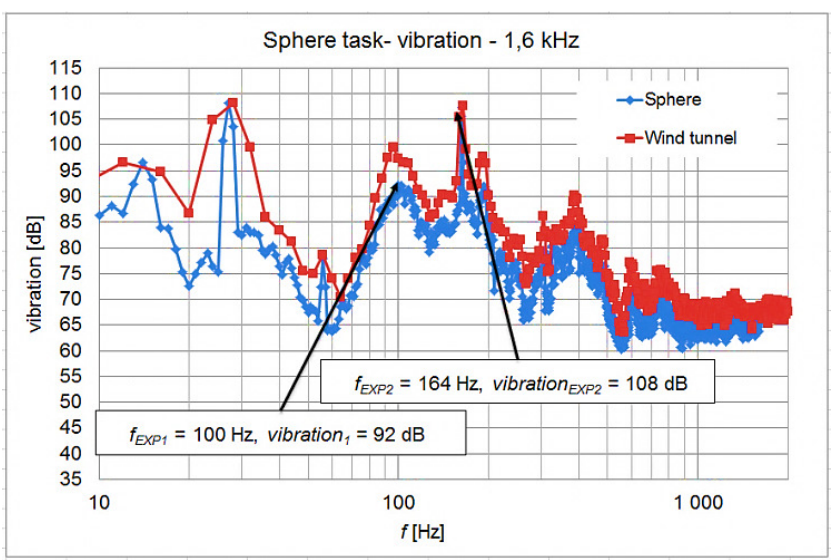

Fig.19. Vibration measurement results for flow around the sphere.

\section{CONCLUSIONS}

This paper presents a complex experimental methodology for aero-acoustic measurement. It consists of two separate measurements that complement each other. In order to verify the proposed experimental measurement methodology, simple tasks of the flow around a sphere and cylinder were 
selected. In the first part, experimental measurements were performed using the hot-wire anemometry method MiniCTA with the 55P14 probe.

The obtained result is a velocity profile of the timeaveraged component velocity $u_{S X}$ at the input to the measuring section for the cylinder, which is graphically illustrated in Fig.3. For the sphere, the resulting profile is shown in Fig.8. The graphical representation of the intensity turbulence measurement results $I_{T S X}$ can be seen in Fig.4. and Fig.9. for the cylinder and sphere, respectively. These measurements serve to determine the boundary conditions of the mathematical simulations using modern numerical methods from the ANSYS Fluent and OpenFOAM programs based on a CFD code. In Fig.5. graphical results of the experimental measurement of the dimensionless speed profiles are depicted behind the flow around the cylinder at a distance of $3 \times D$ and $4 \times D$. In Fig.6. graphical results of the dimensionless turbulence intensity are depicted behind the flow around the cylinder at a distance of $3 \times D$ and $4 \times D$. Fig. 10 . shows the graphical results of the experimental measurement of the dimensionless velocity profiles behind the flow around the sphere at distances of $2 \times D, 3 \times D$. Similarly, Fig. 11 . shows the graphical results of the dimensionless turbulence intensity behind the flow around the sphere at a distance of $2 \times D, 3 \times D$. The main reason of the higher intensity of turbulence on the entrance of the wind tunnel is the combination of construction of the suction part (this part is used from the original wind tunnel) and electrical interference from other electric devices. These results only serve to verify the current field calculated by the CFD methodology using the respective turbulent model. The accuracy of the calculation of the current field strongly influences the subsequent prediction of noise in the area.

Fig.15. shows the results of the experimental SPL sound pressure measurement for the tunnel without an inserted body - the red curve, and the results of the SPL measurements when the flow is around the cylinder in the tunnel are plotted as the blue curve. From these results, it can be seen that the $S P L$ sound pressure level is higher for the flow around the cylinder, for the first peak at $f_{E X P I}=192 \mathrm{~Hz}$ and at the sound pressure level $S P L_{E X P 1}=102 \mathrm{~dB}$. The second peak for the flow around the cylinder has the experimentally obtained parameters $f_{E X P 2}=380 \mathrm{~Hz}$, and $S P L_{E X P 2}=99 \mathrm{~dB}$. From the results for the flow around the sphere task, the first peak is seen at $f_{E X P 1}=100 \mathrm{~Hz}$ and at the sound pressure level $S P L_{E X P I}=92 \mathrm{~dB}$.

The results of the vibro-acoustic measurement, i.e. $S P L$ sound pressure levels measured by the microphone with nose cone, will be used to verify the results from numerical simulations using CFD codes obtained from acoustic analogies, i.e. Curle's analogy (OpenFOAM) and FfowcsWilliams and Hawkings analogy (ANSYS Fluent). A numerical solution of the complex aero-acoustic task can be applied to the real geometry of the means of transport, thus helping to find the optimal shape using modern CAE methods. However, for a good and stable methodology for the numerical simulation solution, the experimental methods for the flow and acoustic fields must be used.

\section{ACKNOWLEDGMENT}

This work was supported by the European Regional Development Fund in the Research Centre of Advanced Mechatronic Systems project, project number CZ.02.1.01/0.0/0.0/16_019/0000867 within the Operational Programme Research, Development and Education.

\section{REFERENCES}

[1] Sun, Z., Song, J., An, Y. (2012). Numerical simulation of aerodynamic noise generated by high speed trains. Engineering Applications of Computational Fluid Mechanics, 6 (2), 173-185.

[2] Détry, S., Manera, J., Detandt, Y., d'Udekem, D. (2010). Aero-acoustic predictions of industrial dashboard HVAC systems. In 24th National Conference on Noise Control Engineering 2010 (NoiseCon 10). Ashland, Ohio: Noise Control Foundation, 870-881.

[3] Manera, J., Detandt, Y., d'Udekem, D., Détry, S. (2009). Aero-acoustic predictions of automotive instrument panel ducts. SAE Technical Paper 2009-012237.

[4] Van Antwerpen, B., d'Udekem, D., Bourachot, J.-L., Leandre, J.-P., Walbott, A., Bouvier, B. (2009). Vibroacoustic simulation of diesel injection ducts. SAE Technical Paper 2009-01-2057.

[5] Gustafsson, M., Jacqmot, J., Caro, S. (2010). Experimental validation of an efficient procedure for large acoustic radiation problems. In Proceedings of ISMA2010 including USD2010. Heverlee, Belgium: Katholieke Universiteit Leuven, 4557-4566.

[6] Dechipré, H., Hartmann, M. (2009) Aeroacoustics simulation of an automotive A-pillar rain gutter. In 4th European Automotive Simulation Conference (EASC 2009). ANSYS, 12 p.

[7] Murad, N.M., Naser, J., Alam, F., Watkins, S. (2006). Computational aero-acoustics of vehicle A-pillar at various windshield RADII. In Fifth International Conference on CFD in the Process Industries. Melbourne, Australia: CSIRO Publishing.

[8] Ask, J., Davidson, L. (2005). The near field acoustics of a generic side mirror based on an incompressible approach. Research Report 2005:5, Division of Fluid Dynamics, Dept. of Applied Mechanics, Dynamics, Chalmers University of Technology, Göteborg, Sweden, 43 p.

[9] Ask, J., Davidson, L. (2006). The sub-critical flow past a generic side mirror and its impact on sound generation and propagation. In 12 th AIAA/CEAS Aeroacoustics Conference (27th AIAA Aeroacoustics Conference). American Institute of Aeronautics and Astronautics.

[10] Caro, S., Ramonda, A. (2006). TBL Noise generated by a simplified side mirror configuration and acoustic transfer through the window: Modelling using Actran and Fluent. In 12th AIAA/CEAS Aeroacoustics Conference (27th AIAA Aeroacoustics Conference). American Institute of Aeronautics and Astronautics. 
[11] Wang, Y., Gu, Z., Li, W., Lin, X. (2010). Evaluation of aerodynamic noise generation by a generic side mirror. International Journal of Mechanical, Aerospace, Industrial, Mechatronic and Manufacturing Engineering, 4 (1), 120-127.

[12] Nouzawa, T., Li, Y., Kasaki, N., Nakamura, T. (2011). Mechanism of aerodynamic noise generated from frontpillar and door mirror of automobile. Journal of Environment and Engineering, 6 (3), 615-626.

[13] Hartmann, M., Ocker, J., Lemke, T., Mutzke, A., Schwarz, V., Tokuno, H., Toppinga, R., Unterlechner, P., Wickern, G. (2012). Wind noise caused by the sidemirror and A-pillar of a generic vehicle model. In 18th AIAA/CEAS Aeroacoustics Conference (33rd AIAA Aeroacoustics Conference). American Institute of Aeronautics and Astronautics.

[14] Jørgensen, F.E. (2002). How to measure turbulence with hot-wire anemometers - a practical guide. Dantec Dynamics A/S, Skovlunde, Denmark, 73 p.

[15] Tůma, J. (2009). Diagnostika strojů. Ostrava, Czech Republic: VŠB - Technická univerzita Ostrava. (in Czech)
[16] Jablonska, J., Mahdal, M., Kozubkova, M. (2017). Spectral analysis of pressure, noise and vibration velocity measurement in cavitation. Measurement Science Review, 17 (6), 250-256.

[17] Lira, I., Grientschnig, D. (2013). A formalism for expressing the probability density functions of interrelated quantities. Measurement Science Review, 13 (2), 50-55.

[18] Uruba, V. (2016). On 3D instability of wake behind a cylinder. In The Application of Experimental and Numerical Methods in Fluid Mechanics and Energy 2016: XX. Anniversary of International Scientific Conference. AIP Conference Proceedings 1745, 020062.

[19] Uruba, V., Pavlik, D., Prochazka, P., Skala, V., Kopecky, V. (2017). On 3D flow-structures behind an inclined plate. In EPJ Web of Conferences: Experimental Fluid Mechanics 2016 (EFM16), Vol. 143, 02137.

Received May 24, 2018 Accepted January 22, 2018 